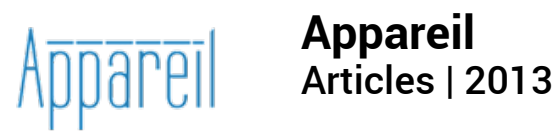

\title{
Simondon et Patočka : les sujets du monde
}

\section{Nicolas Dittmar}

\section{OpenEdition \\ Journals}

\section{Édition électronique}

URL : http://journals.openedition.org/appareil/1741

DOI : 10.4000/appareil. 1741

ISSN : 2101-0714

Éditeur

MSH Paris Nord

\section{Référence électronique}

Nicolas Dittmar, «Simondon et Patočka : les sujets du monde », Appareil [En ligne], Articles, mis en ligne le 26 avril 2013, consulté le 30 juillet 2020. URL : http://journals.openedition.org/appareil/1741 ; DOI : https://doi.org/10.4000/appareil.1741

Ce document a été généré automatiquement le 30 juillet 2020.

\section{(c) (i) (9)}

Appareil est mis à disposition selon les termes de la Licence Creative Commons Attribution - Pas d'Utilisation Commerciale - Pas de Modification 4.0 International. 


\title{
Simondon et Patočka : les sujets du monde
}

\author{
Nicolas Dittmar
}

\section{Introduction}

1 Simondon est le philosophe de l'encyclopédisme génétique, qui représente une tentative majeure pour repenser les fondements de la rationalité à travers l'histoire de la philosophie occidentale : la reprise du thème scolastique de l'individuation, revisitée à la lumière des notions d'information et de transduction montre cette filiation, tout en se démarquant radicalement de tout présupposé substantialiste: l'enjeu est de désubstantialiser l'individu sans le déréaliser, en se fondant sur un réalisme de la relation qui, comme le rappelle Jean-Hugues Barthélémy ${ }^{1}$, est un préalable épistémologique pour comprendre la philosophie génétique de Simondon.

2 En nous appuyant sur ce postulat de base, nous proposons ici d'analyser le rapport de Simondon à Patočka, en montrant comment ces deux penseurs construisent une philosophie post-phénoménologique, qui repose sur le primat du sujet sensible et de sa nature génétique dans le procès de connaissance : comment penser la genèse de l'être, et plus précisément une phénoménologie de l'individuation? L'enjeu de ce questionnement nous semble être de réhabiliter la perception ${ }^{2}$, en portant la méthode de l'épochè phénoménologique à son terme, de l'universaliser en pratiquant une double réduction : celle qui porte sur l'attitude naturaliste et celle qui porte sur le cogito, ce qui revient à dépasser le privilège accordé à la noèse par Husserl dans la constitution du sens.

3 Nous commencerons par examiner le rapport entre l'intentionnalité et l'affectivité, en nous appuyant sur l'analyse simondonienne de la sphère affectivo-émotive, ce qui nous permettra de dégager la dimension prénoétique du sens, et donc de l'intentionnalité. Dans un second temps, nous analyserons la conception de Patočka concernant la méthode de l'épochè, ce qui permettra de voir le lien étroit qui unit cette dernière à la conception simondonienne du préindividuel. Enfin, nous nous demanderons en quoi 
renouveler le sens de la perception permet de comprendre la constitution du sujet, qui se donne comme ego corporel et non plus comme res cogitans.

\section{Le mouvement de l'intentionnalité}

4 La sphère affectivo-émotive possède un sens profond chez Simondon, elle est le retentissement du préindividuel en l'homme ou la forme préindividuelle de l'expérience. Elle se donne dans le temps originel, comme flux héraclitéen des vécus, elle est éminemment transductive : «L'affectivité et l'émotivité seraient alors la forme transductive par excellence du psychisme, intermédiaire entre la conscience claire et la subconscience ${ }^{3} "$.

5 En ce sens, l'affectivo-émotivité définit un stade antérieur à toute pensée : elle serait le lieu des intentions originaires, couples de sensation et de tropisme, orientation du vivant dans un milieu. Simondon nous dit :

Au niveau de l'affectivité et de l'émotivité, la relation de causalité et la relation de finalité ne s'opposent pas : tout mouvement affectivo-émotif est à la fois jugement et action préformée [...]. La polarisation affectivo-émotive [...] est une résultante ou comporte une intentionalité .

6 En effet, l'intentionnalité définit la vie courante des individus, nos sentiments, nos jugements, nos désirs pèsent sur la réalité, notre personnalité s'exerce et se confronte aux autres dans la colère ou la joie, ce que Merleau-Ponty appelle l'«attitude personnaliste ${ }^{5} »$. Autrement dit, nos intentions sont aussi animées par des appréhensions (Auffassung), qui nous amènent à nous dépasser vers les choses et les personnes, dans un contexte vivant. C'est en ce sens que, comme l'a admirablement souligné P. Ricœur :

l'intentionnalité peut être décrite avant et après la réduction phénoménologique: avant, elle est une rencontre, après, elle est une constitution. Elle reste le thème commun de la psychologie pré-phénoménologique et de la phénoménologie transcendantale ${ }^{6}$.

7 Nous pouvons dire que la subconscience est donc une condition de la conscience: « sans l'affectivité et l'émotivité, la conscience paraît un épiphénomène et l'action une séquence discontinue de conséquences sans prémisses ${ }^{7}$ ».

8 Nous qualifierons ici cette condition comme pouvoir de synthèse prénoétique: la subconscience affective et émotive serait cette forme d'intentionnalité primitive qui prête déjà un sens au réel en tant qu'être situé et polarisé par la sphère affectivoémotive, qui est tendance ou tension vers le dehors à partir du centre que définit l'individualité. Selon Patočka, l'intentionnalité peut ainsi être comprise comme Désir, selon une caractérisation dynamique du sujet qui se comprend alors comme «mouvement», dans sa dimension perceptive et sensorielle. Comme le remarque Renaud Barbaras :

Si l'expérience que nous sommes est celle de la liberté, l'être de l'ego consiste en un faire... Ceci revient à reconnaître que la perception, qui délivre l'étant en sa transcendance, doit être comprise comme renvoyant à un mouvement : plus profond que le voir, il y a le se-mouvoir [...] C'est parce que le sujet, en tant que sujet pour le monde, est en son fond désir qu'il ne peut se rapporter au monde que sur le mode de l'agir : le désir est le moteur du mouvement et donc la condition de possibilité de la libertés. 
Si la subconscience affectivo-émotive se conçoit chez Simondon comme mouvement de l'être qui par là même est déjà dans une relation de sens au sein du monde qu'il perçoit, elle permet d'approfondir le thème majeur de la phénoménologie husserlienne, à savoir l'intentionnalité : avant la réduction, il y a cette attitude naturelle qui contient une vérité irréfléchie, et qui se réalise dans l'événement originaire d'une rencontre entre le sujet charnel, animé par un Désir, et le monde des choses. Ce n'est pas l'Esprit qui suit et décrit les phénomènes de corrélation pour constituer le sens des phénomènes en tant qu'ils apparaissent à une conscience. Cette noèse qui exerce et déplie les intentions impliquées dans la subjectivité à partir de la perception des phénomènes n'est possible qu'après la réduction phénoménologique, et est soumise à l'idéal d'adéquation. Pourtant, elle semble escamoter le fait originaire de la liaison sensible et première de l'individu au monde, c'est-à-dire la relation. Patočka partage en ce sens avec Simondon la thèse du réalisme de la relation :

La structure interne du sujet doit d'ores et déjà comporter une corrélation avec les autres étants. Seul un sujet de ce genre peut réaliser des relations. Plus le domaine des relations qu'il est à même de réaliser est varié, plus il est vaste et doté de signification, plus riche sera la vie intérieure du sujet, consistant en l'ouverture à ce domaine [...] Ce dedans le plus originaire se distingue ainsi des relations de type objectif, des relations pures dont les termes sont indifférents... C'est le dedans d'un univers qui connaît des différences plutôt intensives qu'extensives; un dedans qui réside plutôt dans un plus haut degré de dépendance par rapport à d'autres êtres 9 .

\section{2. Épochè et individuation}

Patočka récuse la thèse de l'immanence absolue des vécus subjectifs dans la transparence du cogito, qui conduit à un subjectivisme compromettant l'intentionnalité. Il y a bien une liberté fondamentale du sujet vis-à-vis du donné naturel, qui s'affirme comme autotranscendance, mais cette inspiration libérale se fait à la faveur du monde, s'enracine en lui, selon un mouvement ontogénétique.

11 Le mouvement est un concept central chez Patočka, qui permet de penser la genèse du sujet, en le rapportant à une totalité antérieure à la dualité sujet-objet : «Concept de mouvement comme fondement - mouvement conçu, non pas comme mouvement de l'objet, mais comme œuvre de la physis, avant toute objectivation ou subjectivation - la physis comme essence qui est événement, essence qui advient ${ }^{10} »$.

12 Simondon conçoit l'origine même de la philosophie dans les mêmes termes. Citons ici un passage d'Imagination et invention qui est à ce titre éclairant :

Mais pourtant, le caractère primordial du contenu moteur en toute image d'anticipation a priori, malgré la fixité des archétypes, était latent chez Platon; la philosophie est aussi une connaissance des mixtes, de la dyade indéfinie, de la génésis eis ousian ${ }^{11}$.

13 Si d'ailleurs, Merleau-Ponty critique l'idéalisme husserlien, au nom d'une «foi perceptive » qui nous rattache déjà à l'être et qui représente le lieu de la constitution du savoir, c'est Patočka qui radicalise cette critique et la mène jusque ses ultimes conséquences. Il convient de penser le sujet dans toute sa concrétude, condition de la plénitude du sens visé par l'intentionnalité. Ce sujet est corps, appartenance charnelle au monde. C'est donc dans l'ego corporel qu'il faut chercher les sources de nos intentions, l'intentionnalité pouvant se caractériser chez Patočka, comme nous l'avons $\mathrm{vu}$, comme Désir. 
14 Mais l'apport majeur de la phénoménologie de Patočka est qu'elle conduit à une définition plus radicale de l'épochè phénoménologique: suspension de toute thèse d'existence, elle est acte fondamental de liberté, par où le sujet se délivre, s'émancipe des prétentions de la conscience naturelle à peser sur la réalité - dogmatisme de la doxa - c'est-à-dire au fond de ses jugements qui toujours présupposent l'existence du monde comme donné, alors qu'il devrait être sujet d'étonnement et appréhendé comme transcendant, cette absence d'interrogation définissant la naïveté de l'attitude naturelle.

15 Mais le sujet doit aussi renoncer à la thèse du $\operatorname{cogito}^{12}$, du « je pense " pour être dans le monde, sans le réduire, c'est-à-dire pour éviter toute forme d'abstraction: il faut accomplir une réduction de la réduction, c'est-à-dire mettre à son tour hors circuit la thèse du cogito comme conscience constituante se voulant absolue. Il s'agit par cette radicalisation de l'épochè universelle de reconsidérer le couple individu-milieu (dyade préindividuelle chez Simondon), le monde étant conçu comme totalité préalable, antérieure à l'individuation et à la distinction du sujet et de l'objet opérée par la pensée ${ }^{13}$ :

le monde n'est pas somme, mais totalité préalable. On ne peut pas en sortir, s'élever au-dessus de lui. Le monde est, par tout son être, milieu, à la différence de ce dont il est le milieu. Pour cette raison, il n'est jamais objet. Pour cette même raison, il est unique, indivisible. Toute division, toute individuation est dans le monde, mais n'a pas de sens pour le monde ${ }^{14}$.

16 Citons ici Simondon qui définit cette totalité dans les termes d'une philosophie première :

La véritable philosophie première n'est pas celle du sujet, ni celle de l'objet, ni celle d'un Dieu ou d'une Nature recherchés selon un principe de transcendance ou d'immanence, mais celle d'un réel antérieur à l'individuation ${ }^{15}$.

17 Autrement dit, il y a une relation génétique de l'individu au Tout du monde, que nous révèle l'épochè. Cette relation génétique est une condition d'individuation, qui nous révèle un être comme plus qu'unité et plus qu'identité: "l'être possède une unité transductive, c'est-à-dire qu'il peut se déphaser par rapport à lui-même, se déborder lui-même de part et d'autre de son centre ${ }^{16} »:$ c'est soutenir que l'être est déjà situé et inscrit dans une totalité, qu'il est préindividuel, c'est-à-dire traversé de potentiels, par un mouvement vivant se déployant depuis un terme qui le polarise dès l'origine.

18 Cet a priori universel de l'ego chez Patočka est le monde, comme structure fondamentale par laquelle le sujet rencontre les choses et les individus qui le constituent.

19 C'est pourquoi Patočka développe une phénoménologie dite asubjective, mais toujours fidèle à l'intentionnalité : l'intentionnalité est l'acte de prêter un sens au monde, par l'intermédiaire de ce que Patočka appelle l'aperception :

Que signifie l'aperception si ce n'est la composante de notre vécu, éminemment présente dans la perception elle-même, qui fait qu'il y a là pour nous autre chose, davantage que le vécu comme tel, à savoir l'objet de ce vécu? C'est l'aperception, l'appréhension qui fait que je ne vois pas des tâches vertes, mais les feuilles d'un arbre, que la ligne bleue à l'horizon est une chaîne de montagnes, que le gris moucheté de la surface que voici est la page imprimée d'un livre ${ }^{17}$.

20 La donation de sens - Sinngebung - n'est donc pas seulement quelque noèse, pôle subjectif des intentions d'un moi phénoménologiquement réduit, elle est manifestation ${ }^{18}$ dans et par la rencontre, c'est-à-dire phénomène. C'est soutenir que 
l'intentionnalité prend sa source, non plus dans un cogito, mais dans un corps, qui est le lieu indéfait des moteurs profonds de la personnalité, de ses motifs et exigences vitales.

Il faut donc apprendre à penser, pour Patočka comme pour Simondon, une théorie du sujet sensible, selon le devenir génétique, où le mouvement est dévoilement de l'étant fini selon sa profondeur, selon une "théorie cinétique de la perception». Renaud Barbaras a bien noté cet aspect ontogénétique à l'œuvre dans la philosophie de Jan Patočka, qui le rapproche de Simondon :

L'identification en quoi consiste la subjectivité renvoie à celle accomplie par le mouvement ontogénétique, qui détermine l'étant en unifiant ses déterminations [...] Dire que le sujet fait nécessairement partie du monde, c'est reconnaître qu'il doit être possible de mettre en évidence comme un devenir du sujet ou plutôt un devenir sujet, de décrire l'événement par lequel le sujet se constitue comme tel à partir d'une situation que l'on peut qualifier de pré-subjective ${ }^{19}$.

Dans cette perspective, c'est sans doute à Simondon qu'il revient d'avoir le mieux formulé ce que J.-H. Barthélémy appelle la « réduction au devenir » :

il faut partir de l'individuation, de l'être saisi en son centre selon la spatialité et le devenir, non d'un individu substantialisé devant un monde étranger à lui ${ }^{20}$.

\section{Le problème perceptif : la constitution de l'ego corporel}

La perception est pour Simondon au cœur de la théorie de la connaissance, elle est ce qui atteint les essences génétiques, à travers le divers du sensible :

percevoir est bien prendre à travers; sans ce geste actif qui suppose que le sujet fait partie du système dans lequel est posé le problème perceptif, la perception ne saurait s'accomplir [...] C'est en s'orientant dans cette situation que le sujet peut ramener à l'unité les aspects de l'hétérogénéité qualitative et intensive, opérer la synthèse du divers ${ }^{21}$.

24 Ainsi, la perception ne porte pas sur une forme, elle n'est pas la saisie d'une forme, mais l'invention d'une forme qui modifie la relation du sujet et de l'objet : l'eidos perçu n'est pas une abstraction, ni même une réduction phénoménologique, c'est une polarité, une intensité, une prégnance. La perception est mouvement de l'être dans une coappartenance originaire au monde, elle est ce par quoi le lien génétique à la totalité est justifié ontologiquement.

Simondon prend l'exemple de l'aptitude étonnante de l'enfant à reconnaitre les différentes parties du corps chez les animaux qu'il voit pour la première fois : l'enfant est corporellement engagé dans la perception en fonction de l'émotion - sympathie, peur - provoqué par l'animal. Dans ce sens, ce n'est pas la forme seule de l'animal qui est perçue par l'enfant, mais « son orientation dans l'ensemble, sa polarité qui fait qu'il est couché ou dressé sur ses pattes, qu'il fait face ou fuit, a une attitude hostile ou confiante $^{22} »$.

26 C'est donc par le corps que la perception se produit de manière intensive, par le surgissement d'une forme qui est saisie d'une situation dans ses propriétés expressives. Tel est le sens de la prégnance de la perception chez Simondon :

il est difficile de découvrir le carré comme solution d'un problème perceptif. [...] la figure humaine avec son expression amicale ou hostile, la forme d'un animal avec ses caractères extérieurs typiques, sont aussi prégnants que le cercle ou le carré. 
[...] Percevoir un arbre, c'est voir en lui l'axe qui va des racines à l'extrémité des branches ${ }^{23}$.

C'est donc une réalité complète que nous découvre la perception, à la fois noème, pour reprendre la terminologie de Husserl, qui désigne le perçu comme tel, l'apparence noématique ponctuelle (il s'agit de l'animal tel qu'il est perçu dans un maintenant) ; mais il désigne aussi "l'axe céphalo-caudal», dans le sens idéalement identique de l'animal - eidos.

On peut donc dire que la prégnance définit la vraie forme, c'est-à-dire, pour reprendre Simondon, l'information. Substituer la notion d'information à la notion de forme est la clef du problème perceptif et, à plus forte raison, de la science :

On doit considérer le sujet entier dans une situation concrète, avec les tendances, les instincts, les passions [...] L'intensité d'information suppose un sujet orienté par un dynamisme vital : l'information est alors ce qui permet au sujet de se situer dans le monde. Tout signal reçu possède en ce sens un coefficient d'intensité possible [...] Les formes géométriques prégnantes ne nous permettent pas de nous orienter; elles sont des schèmes innés de notre perception, mais ces schèmes n'introduisent pas un sens préférentiel. C'est au niveau des différents gradients, lumineux, coloré, sombre, olfactif, thermique, que l'information prend un sens intensif, prédominant [...] L'objet est une réalité exceptionnelle ${ }^{24}$.

La question de la perception nous met donc au cœur d'une philosophie génétique, qui récuse la logique de la représentation : c'est du point de vue du perçu, c'est-à-dire de l'apparaître comme tel que le sujet se constitue. La perception est constitution du sens d'être. La constitution de la notion d'objet ne peut se réaliser en escamotant le sujet sensible, qui est le lieu indéfait de sensations et d'impressions impliquées dans l'acte perceptif : la perception a rang d'être, elle est au cœur du mouvement d'individuation. Comme le remarque R. Barbaras,

la difficulté à penser la double condition du sujet provient de la prégnance de l'opposition entre res cogitans et res extensa, qui nous conduit à ne concevoir d'expérience perceptive que transcendant l'être en vie et d'être en vie qu'étranger à l'ordre perceptif [...] Par-delà le vert comme contenu sensible et plus profondément que lui, il y a la signification vitale du vert, le type de rencontre qu'il représente pour l'organisme [...] Autrement dit, saisi du point de vue de l'être vivant qui y est confronté, le perçu possède nécessairement une signification motrice ; en tant que relation d'une totalité organique avec un événement de son milieu, la perception comporte par essence un lien interne avec le mouvement ${ }^{25}$.

S’il revient à Patočka d'avoir le mieux développé cette question du mouvement ontogénétique qui "réalise l'être dont il est le mouvement ", annonçant Simondon, Merleau-Ponty nous a légué une approche décisive du sujet perceptif, qui se rapporte à l'esthésiologie, et qui met en jeu le problème crucial de la recherche d'adéquation entre le perçu, c'est-à-dire l'être-en-vie (Leben), et le ressenti ou l'éprouvé (Erleben), problématique qui est au cœur de la théorie simondonienne de la signification ${ }^{26}$.

Merleau-Ponty conçoit cette recherche comme une "réduction d'écart ", c'est-à-dire comme acte concret de l'individu situé dans un contexte vivant qui procède par «mouvements prospectifs", comme pour sonder le sensible et se faire chose parmi les choses :

Moi qui contemple le bleu du ciel, je ne suis pas en face de lui un sujet acosmique, [...] je ne déploie pas au-devant de lui une idée du bleu qui m'en donnerait le secret, je m'abandonne à lui, je m'enfonce dans ce mystère, il se pense en moi, je suis le ciel même qui se rassemble, se recueille et se met à exister pour soi, ma conscience est engorgée par ce bleu illimité27. 
eut ici, nous semble-t-il, fonder cette plénitude de la perception dans l'« ego corporel » de Patočka, qui considère aussi que la perception s'ancre dans le mouvement de l'existence du sujet, avant même la pure cogitatio :

Le sum n'est pas une chose en ce sens qu'il ne peut jamais apparaître de manière autonome, qu'il ne se manifeste qu'en liaison et en connexion avec des comportements relatifs aux choses. Aussi apparait-il toujours comme ego corporel, aux impulsions duquel le corps propre apparaissant est à même d'obéir. Le corps propre en tant qu'égologique répond à un appel phénoménal, satisfait ou cherche à satisfaire à une exigence, posée par la chose apparaissante, qui s'ouvre devant moi [...] Les possibilités d'action, les occasions, le matériel qui s'offre à moi, m'attirent ou me repoussent. L'attraction ou la répulsion, se déroulant dans le champ phénoménal, requiert une réalisation accomplie par mon corps [...] La réflexion de l'ego doit avoir un caractère tout autre, essentiellement pratique, originant dans l'essence initialement pratique du contexte de notre vie ${ }^{28}$.

\section{Conclusion}

Nous avons vu qu'il existe chez Simondon comme chez Patočka une même démarche fondatrice, qui consiste à penser le sujet polarisé, orienté par une dynamique du sensible, et qui connaît de ce fait des différences intensives : c'est dans le mouvement ontogénétique du sujet que s'élabore la connaissance. Il y a genèse du savoir, et non plus seulement extension déductive ou inductive d'un cogito préalable. C'est récuser l'Idée, au sens kantien, et son schématisme transcendantal, bien que la conception kantienne de l'imagination autorise à maintenir une ouverture à un mode de relation analogique entre la pensée et les choses ${ }^{29}$.

Point d'idéalisme chez les deux auteurs, mais une phénoménologie radicale qui affirme le primat de l'apparaître sur l'être, de l'individuation sur l'individu: de Patočka à Simondon, il y a le sens d'une réduction sans abstraction, reprise du thème husserlien de l'épochè pour l'appliquer à la pensée elle-même, et laisser au monde la possibilité d'apparaître comme tel. Exister dans l'apparition, c'est affirmer la liberté conjointe de l'homme et du monde : l'individuation est alors une condition de la liberté humaine : «à travers l'individu, transfert amplificateur de la Nature, les sociétés deviennent un monde » nous dit Simondon.

\section{BIBLIOGRAPHIE}

Barbaras Renaud, Le désir et la distance. Introduction à une phénoménologie de la perception, Paris, Vrin, 2006.

Barbaras Renaud, Le mouvement de l'existence. Études sur la phénoménologie de Jan Patocka, Paris, La Transparence, 2007.

Barthélémy Jean-Hugues, Simondon ou l'encyclopédisme génétique, Paris, PUF, 2008.

Kant Emmanuel, Critique de la raison pure, Paris, PUF (Quadrige), 2004. 
Merleau-Ponty Maurice, Éloge de la philosophie, Paris, Gallimard (Folio/Essais), 1960.

Merleau-Ponty Maurice, Phénoménologie de la perception, Paris, Gallimard (Tel), 1976.

Patočka Jan, Platon et l'Europe, Lagrasse, Verdier, 1983.

Patočka Jan, Papiers phénoménologiques, Grenoble, Millon (Krisis), 1995.

Patočka Jan, Introduction à la phénoménologie de Husserl, Grenoble, Millon (Krisis), 2002.

Patočka Jan, Qu'est-ce que la phénoménologie ? Grenoble, Millon (Krisis), 2002.

Ricœur Paul, Introduction, in Edmund Husserl, Idées directrices pour une phénoménologie, t. I,

Gallimard, 1950.

Simondon Gilbert, L'individu et sa genèse physico-biologique, Paris, PUF (Épiméthée), 1964.

Simondon Gilbert, L'individuation psychique et collective, Paris, Aubier, 1989.

Simondon Gilbert, L'individuation à la lumière des notions de forme et d'information, Grenoble, Millon

(Krisis), 2005.

Simondon Gilbert, Cours sur la perception, Chatou, La Transparence, 2006,

Simondon Gilbert, Imagination et invention, Paris, La Transparence, 2008.

\section{NOTES}

1. Jean-Hugues Barthélémy, Simondon ou l'encyclopédisme génétique, Paris, PUF, 2008.

2. Simondon apporte un diagnostic qui nous semble fondamental pour comprendre le sens de sa pensée, en examinant les caractères communs des thèses rationalistes et empiristes : "on pourrait dire que la sensation et la perception sont impensables dans un système où précisément l'être est déjà en acte dès le début... : c'est la réception, impliquant extériorité et virtualité qui ne peut être pensée à l'époque classique ». Gilbert Simondon, Cours sur la perception, Chatou, La Transparence, 2006, p. 65.

3. Gilbert Simondon, L'individuation à la lumière des notions de forme et d'information, Grenoble, Millon, 2005, p. 246.

4. Ibid., p. 247-248.

5. « la doxa de l'attitude naturelle est une Urdoxa, elle oppose à l'originaire de la conscience théorique l'originaire de notre existence, ses titres de priorité sont définitifs et la conscience réduite doit en rendre compte ». Maurice Merleau-Ponty, «Le philosophe et son ombre ", in Éloge de la philosophie, Paris, Gallimard (Folio/Essais), 1960, p. 207.

6. Paul Ricœur, Introduction, in Edmund Husserl, Idées directrices pour une phénoménologie, t. I, Paris, Gallimard, 1950, p. 20.

7. Gilbert Simondon, L'individuation à la lumière des notions de forme et d'information, p. 248.

8. Renaud Barbaras, Le mouvement de l'existence. Études sur la phénoménologie de Jan Patočka, Chatou, La Transparence, 2007, p. 26-27. Merleau-Ponty confirme cette dimension fondamentale du mouvement en jeu dans l'intentionnalité : « Toute la connaissance, toute la pensée objective vivent de ce fait inaugural que j'ai senti, que j'ai eu, avec cette couleur ou quel que soit le sensible en cause, une existence singulière qui arrêtait d'un coup mon regard, et pourtant lui promettait une série d'expériences indéfinie, concrétion de possibles d'ores et déjà réels dans les côtés cachés de la chose, laps de durée donné en une fois. L'intentionnalité qui relie les moments de mon exploration, les aspects de la chose, et les deux séries l'une à l'autre, ce n'est pas l'activité de liaison du sujet spirituel, ni les pures connexions de l'ob-jet, c'est la transition que j'effectue 
comme sujet charnel d'une phase du mouvement à l'autre». Maurice Merleau-Ponty, «Le philosophe et son ombre ", in Éloge de la philosophie, p. 212-213.

9. Jan Patočka, Qu'est-ce que la phénoménologie ?, Grenoble, Millon (Krisis), 2002, p. 45-46. Pour Simondon, la méthode consiste à ne pas « essayer de composer l'essence d'une réalité au moyen d'une relation conceptuelle entre deux termes extrêmes, et à considérer toute véritable relation comme ayant rang d'être ", Gilbert Simondon, L'individuation psychique et collective, Paris, Aubier, p. 23-24. Et, poursuit Simondon: «Lorsque la pensée logique et critique, intervenant avant toute ontologie, veut définir les conditions du jugement valide, elle a recours à une certaine conception du jugement, et corrélativement du contenu de la connaissance, de l'objet et du sujet comme termes. Or, antérieurement à tout exercice de la pensée critique portant sur les conditions de la connaissance, il faudrait pouvoir répondre à cette question : qu'est-ce que la relation? C'est une certaine conception de la relation, et en particulier de l'individualité des termes comme antérieurs à la relation qui est impliquée dans une telle théorie de la connaissance. Or, rien ne prouve que la connaissance soit une relation, et en particulier une relation dans laquelle les termes préexistent comme réalités individuées ", Ibid., p. 221.

10. Jan Patočka, Papiers phénoménologiques, Grenoble, Millon (Krisis), 1995, p. 269. C'est sans doute à travers cette formulation que l'on peut rapprocher de façon pertinente, nous semble-t-il, la philosophie de Patočka avec la thèse simondonienne du préindividuel, les deux auteurs faisant prévaloir une condition de totalité génétique comme origine du savoir.

11. Gilbert Simondon, Imagination et invention, Paris, La Transparence, 2008, p. 61-62. En effet, avant le discours sur la chose - Logos, il y a la relation avec la chose, qui est de l'ordre du préindividuel. C'est cet impensé qu'il faut décrire, que la science philosophique doit assumer comme origine de la vérité. Le préindividuel contient bien cependant une Idée-structure, comme le germe donnant naissance au cristal, mais celle-ci est déjà affectée de sensible, elle se comprend comme dyade indéfinie, mixte de sensible et d'intelligible, d'intuition et de concept. C'est soutenir la valeur de l'Esprit comme étant de l'ordre du préindividuel, c'est-à-dire relation constituante, unité dans la genèse : l'Être est dans la genèse et le sens de la philosophie est de penser l'ontogénèse pour comprendre ce qui, en elle, relève de l'esprit, de la réalisation de l'Être comme spiritualité. Tel est, nous semble-t-il, la portée philosophique du monisme génétique de Simondon.

12. "Que se passerait-il si la thèse du soi n'était pas soustraite à l'épochè, si celle-ci était considérée de manière tout à fait universelle ? En opérant une telle épochè, je ne mettrais pas en doute l'indubitable, le cogito qui se pose soi-même. Je m'abstiendrais seulement de faire usage de cette thèse pour ainsi dire automatique, je la mettrais hors circuit en tant que thèse ».

13. Comme le remarque R. Barbaras, « le résidu de l'épochè telle que nous l'avons définie est bien le monde lui-même: ce qui se découvre comme absolu ou apodictique [...] ce n'est pas la conscience, c'est cette Totalité ». Renaud Barbaras, Le désir et la distance. Introduction à une phénoménologie de la perception, Paris, Vrin, 2006, p. 83.

14. Le monde est cet être préindividuel en lequel il n'existe pas de phases, où des ordres de grandeur se côtoient sans médiation, sans faire intervenir la dimension constituante du devenir. Simondon écrit : « au niveau de l'être saisi avant toute individuation, le principe du tiers exclu et le principe d'identité ne s'appliquent pas ", c'est pourquoi la question du sens ne se pose pas encore à l'échelle de l'être préindividuel, qui est indépendant de l'individuation, bien que source de ses potentiels. Comme l'écrit Simondon, «Dans ce cas, l'individu doit être connu non pas abstraitement, mais en remontant à l'individuation, c'est-à-dire en remontant à l'état à partir duquel il est possible de saisir génétiquement l'ensemble de la réalité comprenant l'individu et son complément d'être. Le principe de la méthode que nous proposons consiste à supposer qu'il y a conservation d'être, et qu'il ne faut penser qu'à partir d'une réalité complète ", in L'individu et sa genèse physico-biologique, Paris, PUF (Épiméthée), 1964, p. 73. C'est soutenir le caractère d'autonomie absolue du régime préindividuel, caractère que l'on peut rapprocher du chôrismos 
platonicicien auquel Patočka se réfère pour affirmer la séparation qui met à part de notre réalité, la transcendance vis-à-vis du donné. L'individuation ne modifie pas le monde compris comme totalité préalable, en revanche elle réalise l'Être du devenir, «ce par quoi l'être devient en tant qu'il est, comme être », et c'est en ce sens, nous semble-t-il, que le postulat d'une réalité préindividuelle relève d'une philosophie première, qui réconcilie l'être et le devenir, c'est-à-dire la problématique centrale de toute notre tradition philosophique occidentale.

15. Gilbert Simondon, L'individuation psychique et collective, Paris, Aubier, 2007, p. 137.

16. Ibid., p. 23.

17. Jan Patočka, Introduction à la phénoménologie de Husserl, Grenoble, Millon (Krisis), 2002, p. 84.

18. «Le problème de la manifestation est plus profond, plus fondamental, plus originel que le problème de l'être ", Jan Patočka, Platon et l'Europe, Lagrasse, Verdier, 1983, p. 177.

19. Renaud Barbaras, Le mouvement de l'existence. Études sur la phénoménologie de Jan Patocka, p. 77, 88.

20. Gilbert Simondon, L'individuation psychique et collective, p. 21.

21. Gilbert Simondon, L'individuation à la lumière des notions de forme et d'information, p. 244. C'est nous qui soulignons.

22. Ibid., p. 236.

23. Ibid., p. 236.

24. Ibid., p. 242.

25. Renaud Barbaras, Le Désir et la distance. Introduction à une phénoménologie de la perception, Paris, Vrin, 2006, p. 110-111.

26. Simondon écrit: «Le problème de l'individu est celui des mondes perceptifs, mais le problème du sujet est celui de l'hétérogénéité entre les mondes perceptifs et le monde affectif, entre l'individu et le préindividuel ; ce problème est celui du sujet en tant que sujet; le sujet est individu et autre qu'individu; il est incompatible avec lui-même ", in L'individuation à la lumière des notions de forme et d'information, p. 253.

27. Maurice Merleau-Ponty, Phénoménologie de la perception, Paris, Gallimard (Tel), 1976, p. 248.

28. Jan Patočka, Qu'est-ce que la phénoménologie ?, p. 213-215.

29. Voir Emmanuel Kant, Critique de la raison pure, Paris, PUF (Quadrige), 2004.

\section{RÉSUMÉS}

Cet article se propose d'analyser le rapport philosophique entre deux auteurs emblématiques du courant post-phénoménologique, Simondon et Patočka. Il centre l'analyse sur les présupposés communs aux deux auteurs, tout en développant les fondements d'une phénoménologie de l'individuation. Le postulat majeur commun aux deux auteurs est celui du réalisme de la relation, mais aussi celui de la thèse du préindividuel, qui permet de penser la genèse de l'individu et de la pensée à rebours de tout présupposé substantialiste : il s'agit au fond d'élaborer une nouvelle théorie de la connaissance fondée sur une analytique du sujet sensible, et qui ne déréalise pas l'individu en le faisant éclater dans une pure philosophie de la différence, mais au contraire le révèle dans son être en tant qu'il devient. C'est soutenir la permanence du sujet en amont de toute philosophie de la représentation, permanence du devenir du sujet qui devient en tant qu'il est, selon un mouvement de réalisation ontogénétique qui préserve l'unicité de l'individu à travers le temps. 
INDEX

Mots-clés : individuation, préindividuel, totalité, ontogenèse, relation, épochè, perception

\section{AUTEUR}

\section{NICOLAS DITTMAR}

Docteur en Sciences de l'éducation à l'université de Nantes ndittmar@club-internet.fr 